\title{
Disseminated sarcoidosis resulting in portal hypertension and gastrointestinal bleeding: A rare presentation
}

\author{
Chinedu Ivonye MD FACP, Balsam Elhammali MD, Marshaleen Henriques-Forsythe MD,
}

Raquel Bennett-Gittens MD, Adesoji Oderinde MD FACP

\begin{abstract}
A 41-year-old man was evaluated for an upper gastrointestinal bleed. He was stabilized and an upper esophagogastroduodenoscopy revealed gastritis and esophageal varices. A computed tomography (CT) scan of the abdomen showed hepatosplenomegaly and moderate ascites. Analysis of the ascitic fluid was consistent with portal hypertension. A liver biopsy demonstrated noncaseating granuloma consistent with sarcoidosis, there was no evidence of liver cirrhosis on pathology. Portal hypertension associated with sarcoidosis is rare; after the first reported case in 1949, 35 other cases have been reported in the English literature, with only 16 patients presenting with portal hypertension without evidence of liver cirrhosis. Our patient is among the small group reported to have sarcoidosis-related portal hypertension without evidence of liver cirrhosis. The present case illustrates the importance of recognizing an uncommon manifestation of sarcoidosis
\end{abstract}

\section{CASE PRESENTATION}

A 41-year-old man presented to the emergency department complaining of palpitations and dizziness with exertion for one month. He reported passing dark stools for three weeks and noted decreased appetite. He denied chest pain, cough, dyspnea, orthopnea, paroxysmal nocturnal dyspnea, abdominal pain, nausea, vomiting, diarrhea or weight loss. His medical history included sarcoidosis and steroidinduced diabetes mellitus while on prednisone, glipizide and insulin. The patient migrated from Nigeria 20 years earlier, and denied toxic exposures, use of alcohol, nicotine or recreational drugs.

On examination, he was afebrile with a blood pressure of $121 / 61 \mathrm{mmHg}$, a pulse rate of 93 beats/min, a respiratory rate of 16 breaths/min and oxygen saturation of $98 \%$ on room air. He was anicteric with pale conjunctiva. Respiratory, cardiovascular and neurological examinations were normal. His abdomen was soft, with mild epigastric tenderness but no guarding or rebound tenderness. His liver span was $14 \mathrm{~cm}$ and his spleen extended $4 \mathrm{~cm}$ below the left costal margin. No masses were palpated and bowel sounds were normal. The rectal examination revealed dark stools.

Laboratory studies revealed a white blood cell count of $1.6 \times 10^{9} / \mathrm{L}$, a hemoglobin level and hematocrit of $53 \mathrm{~g} / \mathrm{L}$ and $16.7 \%$, respectively, with a mean corpuscular volume of $68 \mathrm{fL}$, and a platelet count of $6.4 \times 10^{9} /$ L. Electrolytes and coagulation studies were normal, with alanine aminotransferase, aspartate aminotransferase and alkaline phosphatase levels of $37 \mathrm{U} / \mathrm{L}, 27 \mathrm{U} / \mathrm{L}$ and $127 \mathrm{U} / \mathrm{L}$, respectively. He was admitted for symptomatic anemia due to gastrointestinal blood loss and was transfused three units of packed red blood cells. Esophagogastroduodenoscopy revealed gastritis and grade 2, moderately sized esophageal varices that were not actively bleeding. A CT scan

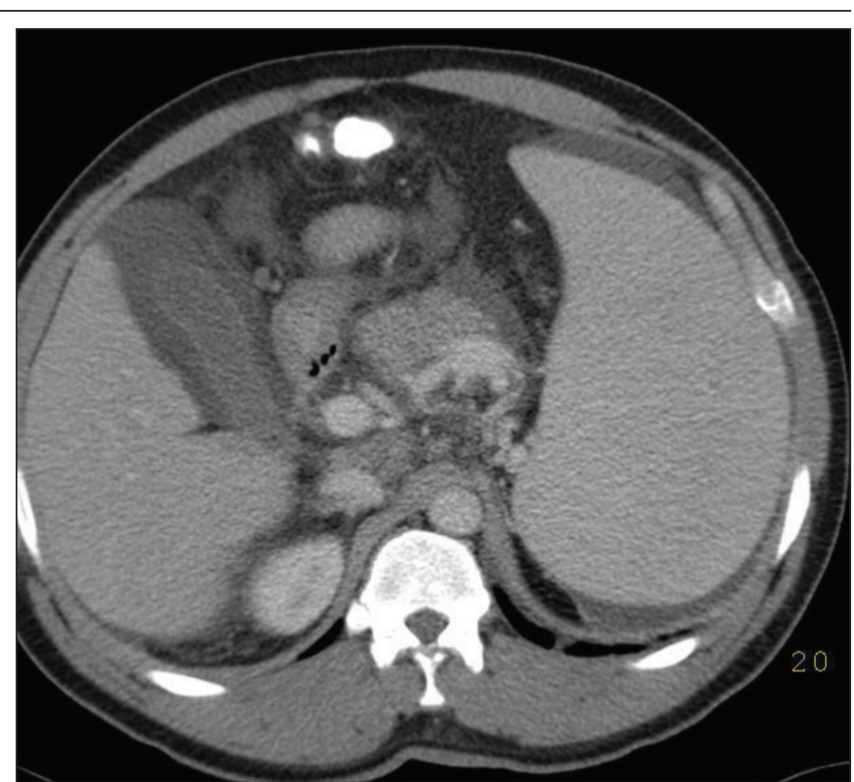

Figure 1) Computed tomography scan showing hepatosplenomegaly

of the abdomen showed hepatosplenomegaly, ascites and thickening of the colonic mucosa with a patent portal sytem (Figure 1). Colonoscopic biopsy of the colonic mucosa was normal. An HIV 1/2 ELISA test was negative and hepatitis serology revealed previous exposure to hepatitis $\mathrm{A}$ and $\mathrm{B}$ viruses with no evidence of chronic infection. Work-up for collagen vascular diseases was negative, and his angiotesin converting enzyme level was within normal limits. Paracentesis revealed strawcoloured ascitic fluid with a serum ascitic albumin gradient of 1.8, consistent with portal hypertension. Liver biopsy showed no evidence of cirrhosis but demonstrated noncaseating granulomas consistent with sarcoidosis (Figure 2). He was started on prophylactic propranolol for recurrence of variceal bleeding. Furosemide and spironolactone were added for management of ascites. The patient declined splenectomy.

\section{DISCUSSION}

Sarcoidosis is a systemic disorder of unknown etiology characterized by noncaseating granulomas with a propensity for several body systems. Lung involvement is most common but liver involvement occurs in approximately $70 \%$ of cases. Patients with hepatic sarcoidosis are typically asymptomatic with normal liver enzyme levels. Hepatic granulomas are found on CT imaging in $<5 \%$ of patients and are typically

Department of Internal Medicine, Morehouse School of Medicine, Atlanta, Georgia, USA

Correspondence: Dr Chinedu Ivonye, Department of Internal Medicine, Morehouse School of Medicine, 720 Westview Drive Southwest, Atlanta,

Georgia 30310, USA. Telephone 404-616-7085, fax 404-616-7773, e-mail civonye@msm.edu

Received for publication December 27, 2011. Accepted December 30, 2011 


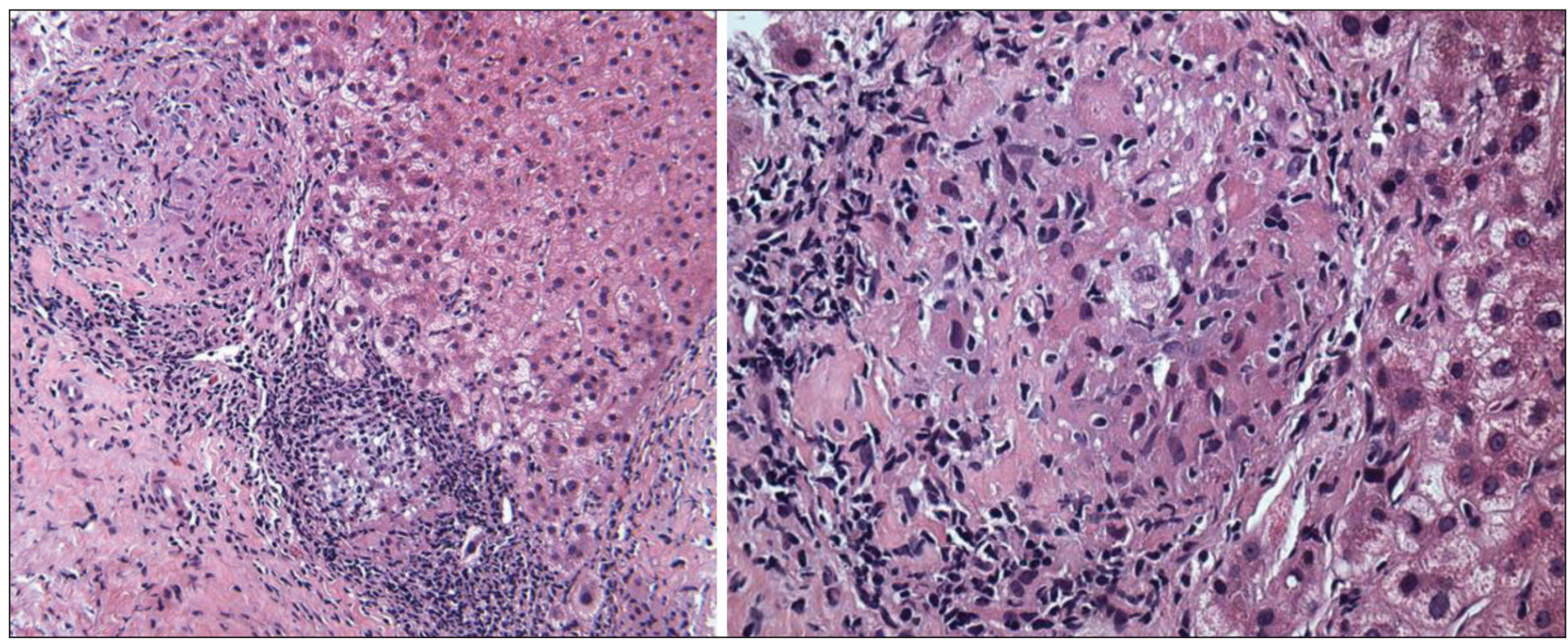

Figure 2) Histopathological specimens of liver biopsy showing a confluent mass of granulomas that have coalesced. Multiple multinucleated giant cells are present. Hematoxylin and eosin stain, original magnification $\times 40$

between $0.5 \mathrm{~cm}$ to $0.8 \mathrm{~cm}$ in size. Clinical manifestations identified include jaundice, chronic cholestasis, portal hypertension, BuddChiari syndrome, and intrahepatic cholestasis resembling primary biliary or sclerosing cholangitis (1).

Portal hypertension is defined by a portal pressure gradient $>11 \mathrm{mmHg}$ or the presence of esophageal varices, first reported in association with sarcoidosis by Mino et al (2) in 1949. Between 1949 and 2001, an additional 35 cases were reported in the English literature (16 had portal hypertension without evidence of cirrhosis). Several mechanisms have been postulated to explain the pathophysiology of portal hypertension related to sarcoidosis. Maddrey et al (3) proposed that arterial-venous shunts within granulomas in the liver and spleen cause elevated portal blood flow resulting in a compensatory increase in intrahepatic resistance. The resistance in the intrahepatic sinusoids may also increase due to obstruction from confluent sarcoid granulomas. Another proposed mechanism is that presinusoidal obstruction by granulomas in the portal vein cause an increase in pressure and restrict flow $(4,5)$. A third theory is that granulomatous phlebitis in portal and hepatic veins leads to cirrhosis and focal fibrosis, which then increases pre- and postsinusoidal resistance (6).

The present patient is among a small group reported to have sarcoidosis-related portal hypertension. His portal hypertension resulted in variceal bleeding that presented as symptomatic anemia. Hypersplenism was likely a consequence of his portal hypertension and may have been responsible for his leukopenia and thrombocytopenia.
Our patient's angiotensin-converting enzyme level remained normal throughout his hospital course. This is not unusual because angiotensinconverting enzyme levels are elevated in only $60 \%$ to $70 \%$ of patients with sarcoidosis and do not correlate well with disease activity. In patients with portal hypertension, splenectomy or insertion of a portocaval shunt has been shown to reduce portal pressures and offer symptomatic relief. Steroids are of no benefit in sarcoidosis-induced portal hypertension.

The present case illustrates the importance of recognizing an uncommon presentation of sarcoidosis.

\section{REFERENCES}

1. Karagiannidis A, Karavalaki M, Koulaouzidis A.

Hepatic sarcoidosis. Ann Hepatol 2006;5:251-6.

2. Mino RA, Murphy AI Jr, Livingstone RG. Sarcoidosis producing portal hypertension; treatment by splenectomy and splenorenal shunt. Ann Surg 1949;130:951-7.

3. Maddrey WC, Johns CJ, Boitnott JK, Iber FL. Sarcoidosis and chronic hepatic disease: A clinical and pathologic study of 20 patients. Medicine 1970;49:375-95.

4. Mistilis SP, Green JR, Schiff L. Hepatic sarcoidosis with portal hypertension. Am J Med 1964;36:470-5.

5. Ishak KG. Sarcoidosis of the liver and bile ducts Mayo Clin Proc 1998;73:467-72.

6. Blich M, Edoute Y. Clinical manifestations of sarcoid liver disease. J Gastroenterol Hepatol 2004;19:732-7.

The Canadian Journal of Gastroenterology is now considering a limited number of submissions for IMAGE OF THE MONTH. These are based on endoscopic, histological, radiological and/or patient images, which must be anonymous with no identifying features visible. The patient must consent to publication and the consent must be submitted with the manuscript. All manuscripts should be practical and relevant to clinical practice, and not simply a case report of an esoteric condition. The text should be brief, structured as CASE PRESENTATION and DISCUSSION, and not more than 700 words in length. A maximum of three images can be submitted and the number of references should not exceed five. The submission may be edited by our editorial team.

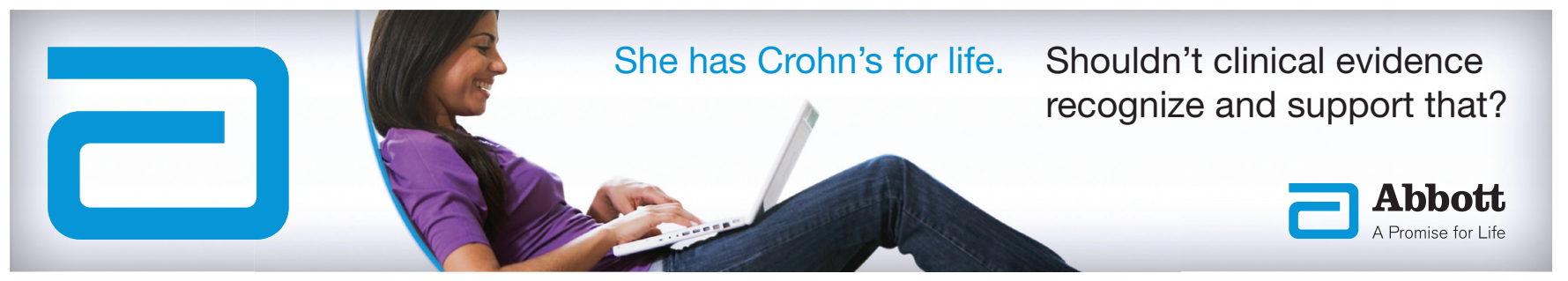




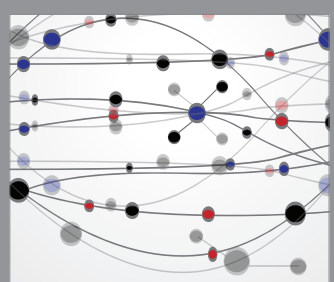

The Scientific World Journal
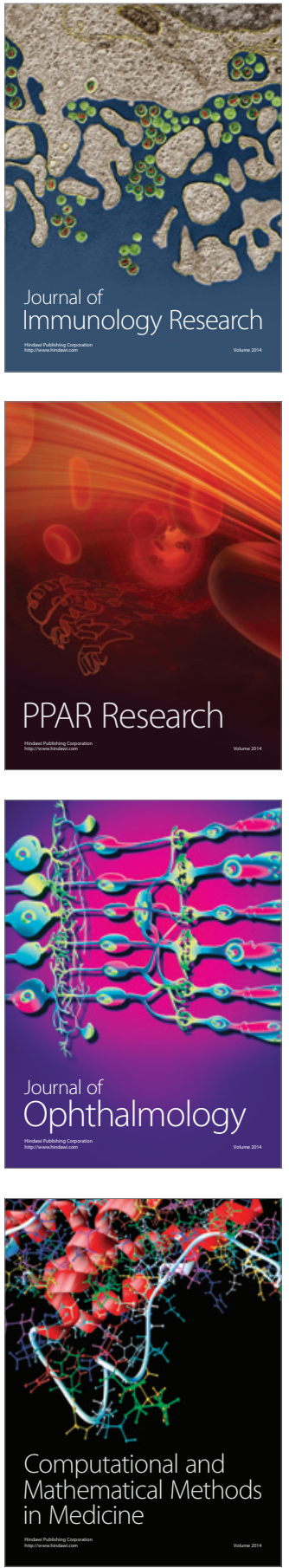

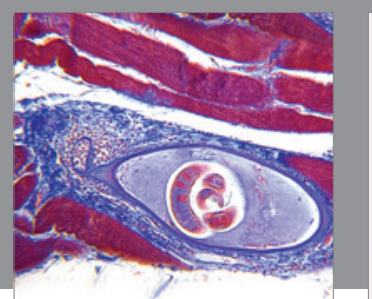

Gastroenterology Research and Practice

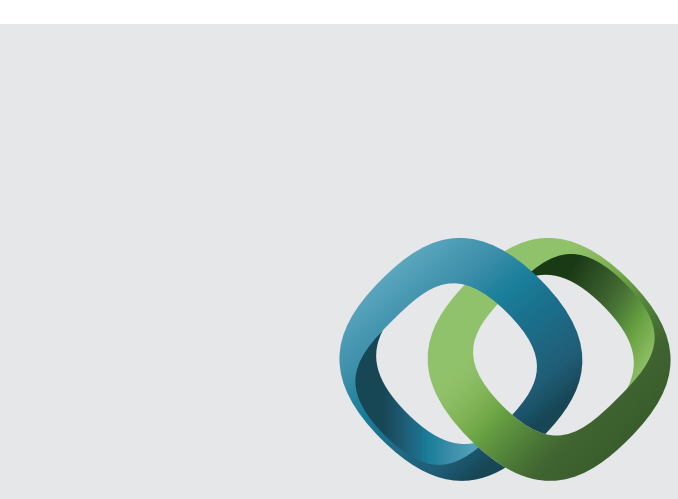

\section{Hindawi}

Submit your manuscripts at

http://www.hindawi.com
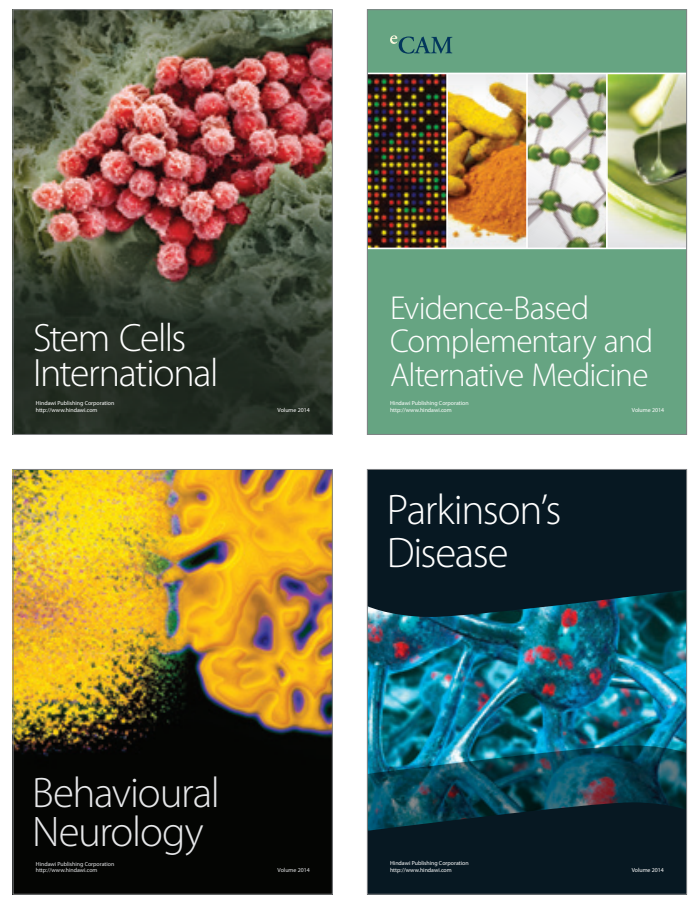
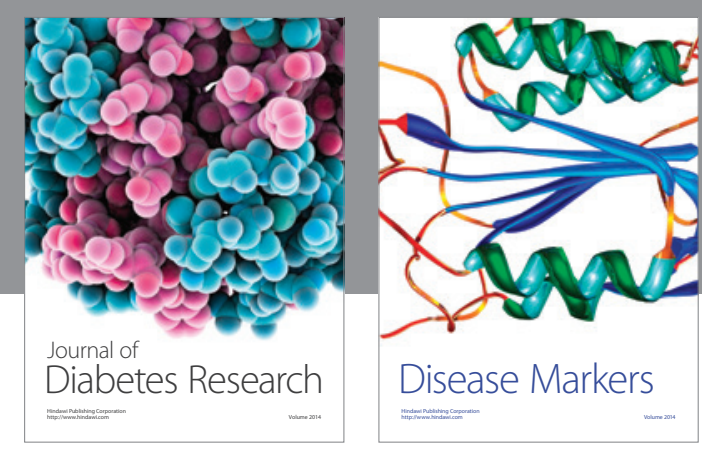

Disease Markers
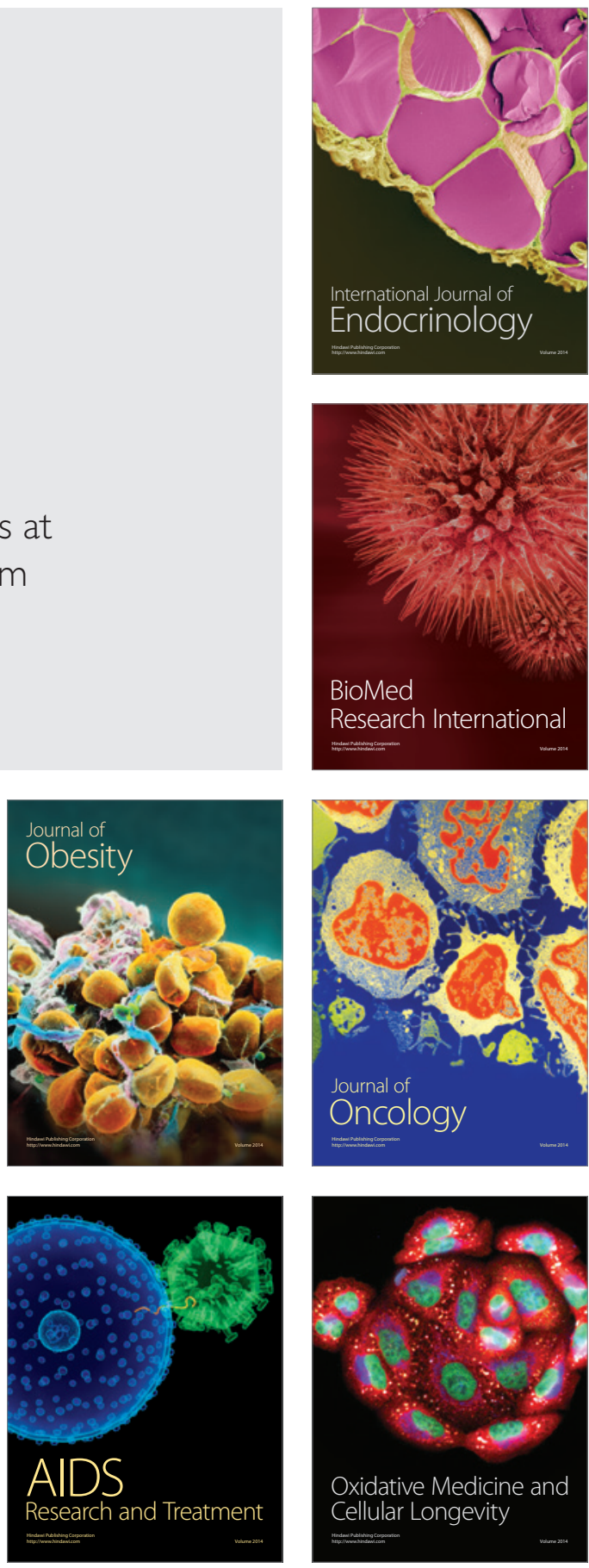